\title{
Investigation of the Water Draining Process Pushed by Gas for U-Type and Z-Type Manifolds
}

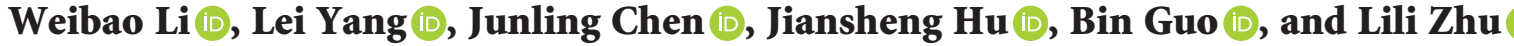 \\ Institute of Plasma Physics, HFIPS, Chinese Academy of Sciences, Hefei 230031, China \\ Correspondence should be addressed to Lili Zhu; lilizhu@ipp.ac.cn
}

Received 2 March 2021; Revised 26 November 2021; Accepted 24 December 2021; Published 21 January 2022

Academic Editor: Arkady Serikov

Copyright (c) 2022 Weibao Li et al. This is an open access article distributed under the Creative Commons Attribution License, which permits unrestricted use, distribution, and reproduction in any medium, provided the original work is properly cited.

\begin{abstract}
In the experimental advanced superconducting tokamak (EAST), the cooling channels of plasma-facing components (PFCs) are familiarly connected in parallel through manifolds. According to the drainage performance of the PFCs, the amount of water trapped in the cooling channels is directly correlated with the type of manifolds. To date, manifold types have been well studied with respect to single-phase and mixed multiphase flow characteristics. However, there are few studies on the drainage performance relevant to the type of manifolds. The friction effect and inertia effect in the manifold intake and exhaust are studied through theoretical analysis. In addition, the draining liquid effect in the branches is dependent on the pressure difference and the resistance coefficient. Furthermore, U-type and Z-type manifolds are studied with FLUENT to discuss their drainage capability in this study. The distribution of the water volume fraction, pressure, and flow ratio is obtained to verify that the $Z$ type is more effective than the $U$ type when applied in the drainage process. This is also supported by comparing the experimental value of the two in drainage discharge. This study will provide a theoretical basis for future upgrades of EAST drainage systems.
\end{abstract}

\section{Introduction}

Manifolds are essentially used to supply or return cooling water for plasma-facing components (PFCs), such as divertors, in the experimental advanced superconducting tokamak (EAST). In the process of device maintenance, water filled in the channel needs to be blown out of the PFC cooling channels by pressurized nitrogen through the manifolds. According to the experimental results, the cooling water filled in the single divertor module can be well drained. However, when the cooling channel is connected parallel to the manifolds, water can be trapped in the cooling channel of the PFCs. Therefore, the drainage performance is the most affected by the manifold structure type: the U-type and Z-type manifolds are shown in Figure 1. Manifolds with inlets and outlets on the same side are called U-type manifolds. In contrast, those with the inlet and outlet on the opposite side are called Z-type manifolds. Extensive research has been carried out on the type of manifold structure [1-5]. The results are briefed below.
Miao Zhengqing (2006) obtained analytical solutions by introducing derived continuous and discrete mathematical models for single-phase flow in parallel tube platen systems. The flow characteristics along the header axes and the connecting tubes were studied. When the friction loss in the headers could not be neglected, a corrected velocity to static pressure conversion coefficient would be derived. It can be used to obtain analytical solutions of the corrected static pressure and velocity in headers and connecting tubes [1].

Junye Wang (2008) developed an analytical model based on mass and momentum conservation to solve the flow and pressure distribution in fuel cell stacks with a U-type arrangement. Parameter sensitivity is also studied to determine the influence of geometrical structures and parameters on the flow performance of fuel cell stacks. The solution results show that friction and momentum effects work in opposite directions. A proper balance of the two effects can result in less nonuniformity and an optimal design of U-type fuel cell stacks [2]. 


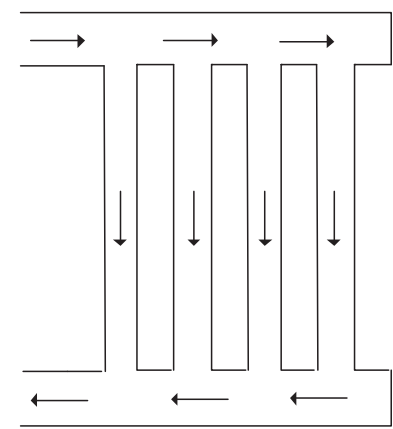

(a)

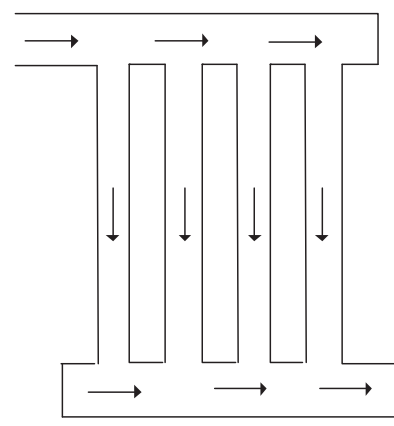

(b)

Figure 1: Manifold type. (a) U type. (b) Z type.

Mayurkumar S. Gandhi et al. (2011) carried out CFD simulations for the flow and pressure distribution of pure steam in header and tube assemblies. The effects of design parameters have been investigated over a wide range, such as the tube pitch, header diameter, tube diameter, number of tubes, and inlet or outlet pipe diameter. Meanwhile, experimental research has been performed on the scaled-down geometry of header configuration "C1." The numerical and experimental results showed good agreement in that the tube diameter, number of tubes, and locational arrangement with respect to the inlet and/or outlet pipe were the most important design parameters affecting the flow and pressure distribution in the pipeline network [3].

Hossein Asgharian et al. (2020) applied a CFD code to investigate the pressure drop in gas channels, including inlet and outlet manifolds with U- and Z-type arrangements, in terms of a 10-cell PEM fuel cell stack. It was demonstrated that there was no significant difference in pressure drop between $\mathrm{U}$ - and Z-type arrangements when dry oxidant and fuel were used. However, when the oxidant gas, especially air, is humidified, utilization of a Z-type arrangement is preferable because of a lower pressure drop [4].

Osman K. Siddiqui et al. (2020) and Simin performed experimental and numerical investigations on the flow distribution in U- and Z-type manifolds. In this study, flow distribution measurements were performed using the particle image velocimetry (PIV) technique in two types of rectangular manifolds. The experimental results were further verified against the results obtained from numerical modeling with similar trends. The results showed a similar flow distribution at a lower flow rate. At a higher flow rate, the U-type manifolds had a better flow distribution than the Z-type manifolds [5].

From the literature review, it is observed that the applicability of different types of manifolds will change according to application conditions. Therefore, it is necessary to analyze the applicability of manifold types under drainage and drying conditions. Moreover, research on the phenomenon of drainage in U-type and Z-type manifolds is rare. In this study, the influence of manifold type on the drainage and drying processes of internal components is analyzed in detail with FLUENT software. The distribution of the water volume fraction, pressure, and flow ratio is obtained to verify that the drainage and drying effects of manifolds are related to the type of manifolds. Furthermore, the results obtained in this study can provide a reference for the design and analysis of fusion reactor drying systems.

\section{Mathematical Analysis}

In the theoretical analysis of manifolds, the mass and momentum conservation equations are applied to the manifold control volume. Unlike single-phase flow, gas-liquid twophase flow is analyzed based on the gas-liquid flow pattern and other assumptions [6-8]. Therefore, the development of the theoretical model is based on the following assumptions [7]:

(1) The gas-liquid two-phase flow pattern in the intake and exhaust manifolds is stratified flow, and it in the manifold branch is annular flow

(2) The two-phase flow in the adiabatic state is stable and uniform in circumference

(3) Gas and liquid in the manifold branch flow in the same direction

2.1. Drainage Characteristics of Branch Pipe. The manifold branch between the manifold intake and exhaust was modeled as shown in Figure 2. The momentum conservation equations of the gas and annular liquid in the branch are applied to the manifold control volume under the separated flow model [9-15].

For the branch of gas flow from the intake shown in Figure 2(a), the momentum conservation equations of the gas and liquid are written as follows:

$$
\begin{array}{r}
\frac{\mathrm{dP}_{b s}}{\mathrm{~d} y} \alpha S_{b}+P e_{i} \tau_{i}-\rho_{g} g \alpha S_{b}=0, \\
\frac{\mathrm{d} P_{b s}}{\mathrm{~d} y}(1-\alpha) S_{b}-P e_{i} \tau_{i}+P e_{w} \tau_{w}-\rho_{l} g(1-\alpha) S_{b}=0 .
\end{array}
$$

Adding equations (1) and (2) together, equation (3) can be obtained as follows:

$$
\frac{\mathrm{d} P_{b s}}{\mathrm{~d} y}=-\frac{4}{D_{b}} \tau_{w}+\rho_{g} g \alpha+\rho_{l} g(1-\alpha) .
$$




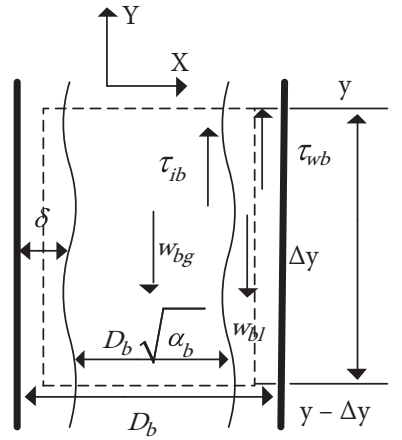

(a)

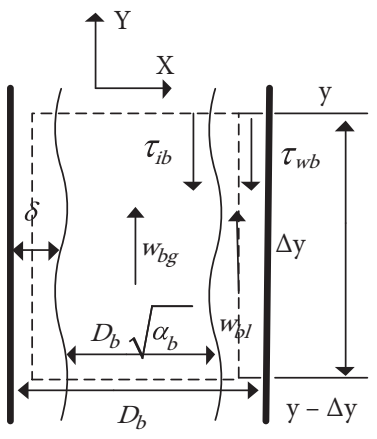

(b)

Figure 2: The theoretical model of the manifold branch. (a) The branch that gas flows from the intake. (b) The branch that gas flows into the exhaust.

After substituting equation (15) into equation (3), equation (3) can be rearranged as the following equation:

$$
\frac{\mathrm{d} P_{b s}}{\mathrm{~d} y}=-\frac{f_{w} \rho_{l}}{2 D_{b}} \cdot u_{l}^{2}+\rho_{g} g \alpha+\rho_{l} g(1-\alpha)
$$

where $\mathrm{d} P_{b s} / \mathrm{d} y$ is the axial pressure gradient of the branch connected through the supply header, $P e_{i}$ and $P e_{w}$ are the wetted perimeter of the pipe wall and circumference of the gas core, respectively, $\alpha$ is the void fraction, $D_{b}$ is the diameter of the manifold branch, $S_{b}$ is the cross-sectional area of the manifold branch, $\tau_{w}$ is the wall shear force, and $\tau_{i}$ is the gas-liquid shear force.

Two constants are defined: A is the resistance coefficient, and $\mathrm{B}$ is the gravity coefficient.

$$
\begin{aligned}
& A=\frac{f_{w} \rho_{l}}{2 D_{b}}, \\
& B=\rho_{g} g \alpha+\rho_{l} g(1-\alpha) .
\end{aligned}
$$

Equation (18) can be rearranged as the following equation:

$$
\frac{\mathrm{d} P_{b s}}{\mathrm{~d} y}=-A \cdot u_{l}^{2}+B
$$

Similar to the deduction of the formula for the branch in which gas flows from the intake, the pressure distribution of the branch in which gas flows into the exhaust can be obtained along the Y-axial coordinate.

$$
\frac{\mathrm{d} P_{b r}}{\mathrm{~d} y}=-A \cdot u_{l}^{2}-B
$$

The pressure gradient can also be expressed as follows:

$$
\begin{aligned}
& \frac{\mathrm{d} P_{b s}}{\mathrm{~d} y}=P_{b s}(y-\Delta y)-P_{b s}(y), \\
& \frac{\mathrm{d} P_{b r}}{\mathrm{~d} y}=P_{b r}(y)-P_{b s}(y-\Delta y) .
\end{aligned}
$$

Neglecting the pressure drop between the branch in which gas flows from the intake and the branch in which gas flows into the exhaust, equations (6) and (7) are added together. Then, substituting equations (8) and (9), equation (10) can be obtained as follows:

$$
P_{b r}(y)-P_{b s}(y)=-2 A u_{l}^{2} .
$$

The relationship of liquid velocity to the branch pipe can be described as follows:

$$
u_{l}=\sqrt{\frac{P_{b r}(y)-P_{b s}(y)}{-2 A}} .
$$

The liquid flow velocity in the branch pipe is mainly directly proportional to the pressure difference between the manifold intake and exhaust and inversely proportional to the resistance coefficient in the branch pipe. In this regard, the following modeling and analysis are carried out to determine the pressure distribution characteristics of the header of the U-type and Z-type manifolds.

2.2. Pressure Distribution Characteristics of the Header. In this study, the unit control volume model of the manifold intake, as shown in Figure 3, is established. The positive $X$ direction is the gas flow direction; the gas-liquid two-phase flow is in a stratified flow state, the gas phase is above the manifold intake, and the liquid phase is below the manifold intake. The gas pressure in the manifold header is the key driving force for drainage. In this way, this study establishes the mass conservation equation and momentum conservation equation for the gas in the manifold intake, as shown in equations [7-13].

\subsubsection{Mass Conservation.}

$$
\begin{aligned}
\rho_{g} F_{g s} U_{g s} & =\rho F_{g s}\left(U_{g s}+\frac{\mathrm{d} U_{g s}}{\mathrm{~d} X} \Delta X\right)+\rho_{g} F_{g b} W_{g b}, \\
W_{g b} & =-\frac{F_{g s} \Delta X}{F_{g b}} \frac{\mathrm{d} U_{g s}}{\mathrm{~d} X}
\end{aligned}
$$

where $F_{g s}$ and $F_{g b}$ are the gas cross-sectional areas of the manifold intake and branch, respectively, $U_{g s}$ is the 


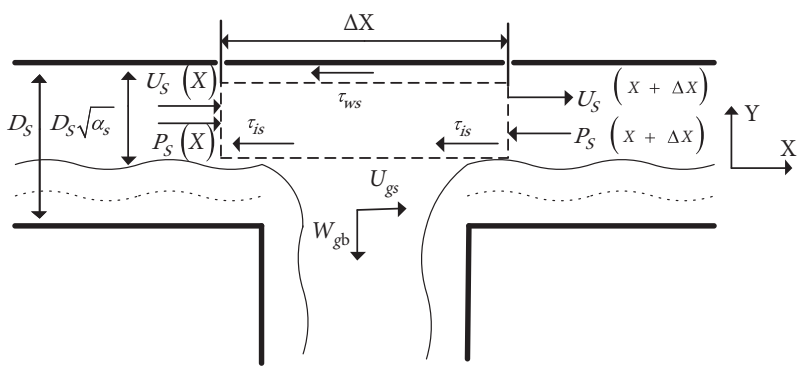

Figure 3: The theoretical model of manifold intake.

manifold intake velocity, $W_{g b}$ is the manifold branch velocity, $\Delta X$ is the length of the manifold intake control 2.2.2. Momentum Conservation. volume, and $\rho$ is the gas density [7].

$$
\begin{aligned}
P_{s} F_{g s}-\left(P_{s}+\frac{\mathrm{d} P_{s}}{\mathrm{~d} X} \Delta X\right) F_{g s}-\left(\tau_{w s g}+\tau_{i s g}\right) \pi D_{s} \sqrt{\alpha_{s}} & =\rho_{g} F_{g s}\left(U_{g s}+\frac{\mathrm{d} U_{g s}}{\mathrm{~d} X} \Delta X\right)^{2}+\rho_{g} F_{g b} U_{g s} W_{g b}-\rho_{g} F_{g s} U_{g s}^{2}, \\
\tau_{w s g} & =\frac{f_{w} \rho_{g} U_{g s}^{2}}{8} \\
\tau_{i s g} & =\frac{f_{i} \rho_{g}\left(U_{g s}-U_{l s}\right)^{2}}{2}, \\
W_{g b} & =\beta_{s} U_{g s} .
\end{aligned}
$$

After substituting equations (13), (15), (16), and (16) into equation (14), equation (14) can be rearranged as the following equation:

$$
\frac{\mathrm{d} P_{s}}{\mathrm{~d} X}=-\frac{f_{w}}{2 D_{s} \sqrt{\alpha_{s}}} \rho_{g} U_{g s}^{2}-\frac{f_{i}}{2 D_{s} \sqrt{\alpha_{s}}} \rho_{g}\left(U_{g s}-U_{l s}\right)^{2}-\left(2-\beta_{s}\right) \rho_{g} U_{g s} \frac{\mathrm{d} U_{g s}}{\mathrm{~d} X},
$$

where $U_{g s}$ and $U_{l s}$ are the gas and liquid velocities of the manifold intake, respectively, $P_{s}$ is the manifold intake pressure, $f_{w}$ is the resistance coefficient between the gas and wall, $f_{i}$ is the resistance coefficient between the gas and liquid, $D_{s}$ is the diameter of the manifold intake, $\alpha_{s}$ is the void fraction of the manifold intake, and $\beta_{s}$ is the average velocity ratio in the manifold intake.
At the same time, this study also establishes the unit control volume model of the manifold exhaust, as shown in Figure 4. The positive direction of $X$ is still right, but the gas flow direction is the $X$ negative direction. For the gas in the manifold exhaust, the mass conservation equation and momentum conservation equation are established. Similarly, the formula in the same form as equation (18) can be obtained, as shown in the following equation:

$$
\frac{\mathrm{d} P_{r}}{\mathrm{~d} X}=\frac{f_{w}}{2 D_{r} \sqrt{\alpha_{r}}} \rho_{g} U_{g r}^{2}+\frac{f_{i}}{2 D_{r} \sqrt{\alpha_{r}}} \rho_{g}\left(U_{g r}-U_{l r}\right)^{2}-\left(2-\beta_{r}\right) \rho_{g} U_{g r} \frac{\mathrm{d} U_{g r}}{\mathrm{~d} X},
$$

where $U_{g r}$ and $U_{l r}$ are the gas and liquid velocities of the manifold exhaust, respectively, $P_{r}$ is the manifold exhaust pressure, $D_{r}$ is the diameter of the manifold exhaust, $\alpha_{r}$ is the void fraction of the manifold exhaust, and $\beta_{r}$ is the average velocity ratio in the manifold exhaust. 


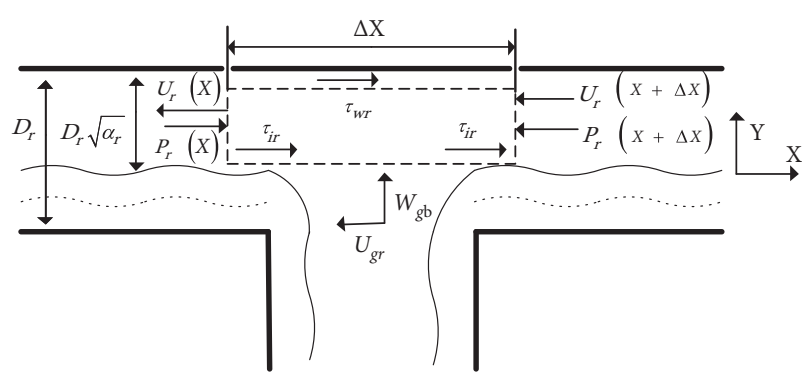

FIgURE 4: The theoretical model of U-type manifold exhaust.

The first two terms of equations (18) and (19) are the friction resistance terms generated by the gas-liquid interphase friction and the friction between the gas and wall [10-15]. The resistance direction is opposite to the flow direction. Therefore, the friction resistance term of equation (18) is a negative term and that of equation (18) is a positive term; the last terms of equations (18) and (19) are the inertia terms caused by the action of diversion and confluence, respectively.

The manifold intake has the function of shunting; the flow gradually decreases along the positive direction of the $X$ axis, and the velocity gradient is less than zero. It is concluded that the inertia term of the last term of equation (18) is positive, but the first two terms are negative. Therefore, the pressure distribution of the manifold intake is mainly the game between the friction resistance term and the inertia term $[15,16]$. The friction term tends to decrease the pressure, and the inertia term tends to increase the pressure. As shown in Figure 5, under ideal working conditions, if the inertia term is dominant, the pressure of the manifold intake gradually increases along the positive $X$ direction (area 1). If the friction resistance term is dominant, the pressure of the manifold intake gradually decreases along the positive $X$ direction (area 3). Under actual working conditions, the pressure distribution of the manifold intake is mainly in shadow area 2 [16-18].

In the manifold exhaust, since the velocity gradient of the manifold exhaust along the positive direction of the $X$ axis is negative, it is concluded that the inertia term is positive. In addition, although the frictional resistance term is negative along the flow direction, it is also positive along the positive direction of the $X$ axis. Therefore, both the inertia term and the frictional resistance term tend to increase the pressure along the positive direction of the $X$ axis. Therefore, the pressure distribution of the manifold exhaust gradually increases along the positive direction of the $X$ axis. As shown in Figure 5, the manifold exhaust outlet of Z-type manifolds is the same as the positive $X$ direction, so the manifold exhaust pressure of the Z-type manifolds gradually decreases along the positive $X$ direction. However, the U-type manifold outlet direction of the manifold exhaust is opposite to the positive $X$ direction, so the manifold exhaust pressure of the U-type manifolds gradually increases along the positive $X$ direction. In short, due to the confluence effect of the manifold exhaust, along the flow direction, the flow velocity increases, the dynamic pressure increases, and the pressure of the collecting main pipe gradually decreases.

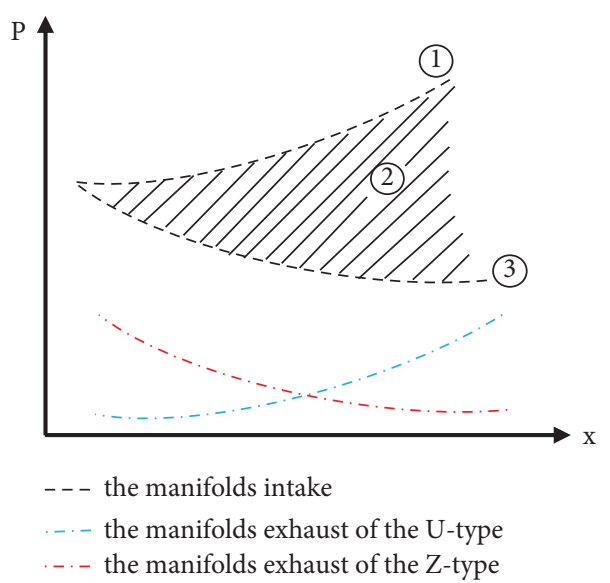

FIGURE 5: Header pressure distribution of different manifold types.

According to the above analysis, the discharge speed of the branch pipe is related to the pressure distribution of the header of the manifolds. Therefore, the pressure distribution of the header caused by the U-type manifolds and Z-type manifolds will inevitably have an important impact on the drainage and drying processes.

\section{CFD Numerical Analysis}

CFD numerical simulation supports flow field analysis, calculation, and prediction so that we can more deeply understand the mechanism of the problem and provide guidance for the experiment $[19,20]$. This part includes the development of the model, mathematical models, and meshing that are applied during the analysis. They are briefly described below [21].

3.1. Model Description. The manifold is composed of the manifold intake (supply header), manifold exhaust (return header), and manifold branches in isometric arrangement (Figure 6). In addition, the left port of the supply header is set as the gas inlet, and the dead ends are set at the right port. Furthermore, the left port of the return header is required to be the outlet in the U-type manifold. The outlet of the Z-type manifold is the right port of the return header.

To track the thermal and hydraulic characteristics of the manifold, monitoring planes are selected along the gravity direction of the manifold (Figure 7).

3.2. Mathematical Models. The VOF model adapted in the research can track the interface of the gas-liquid two-phase flow [21]. Furthermore, the $k-\varepsilon$ turbulence model can accurately predict the phenomenon of shear flow [22], vortex, and separated flow $[21,23]$, including the dissipation rate. In addition, compared the $k-\varepsilon$ turbulence model with the Reynolds stress model [21, 24], the $k-\varepsilon$ turbulence model consumes fewer computing resources [21]. Therefore, the $k-\varepsilon$ turbulence model written as equations (20) and (21) [21] is adopted in this study. 


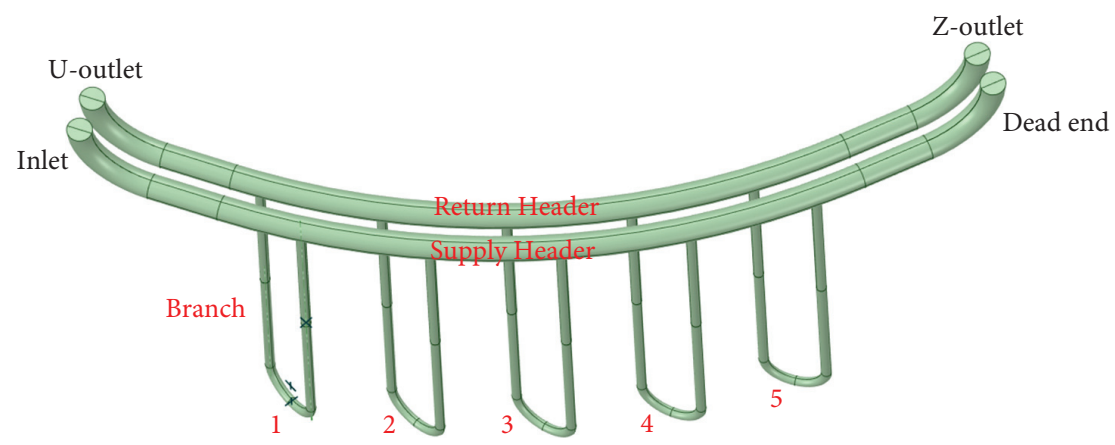

FIGURE 6: Schematic representation of the manifold.

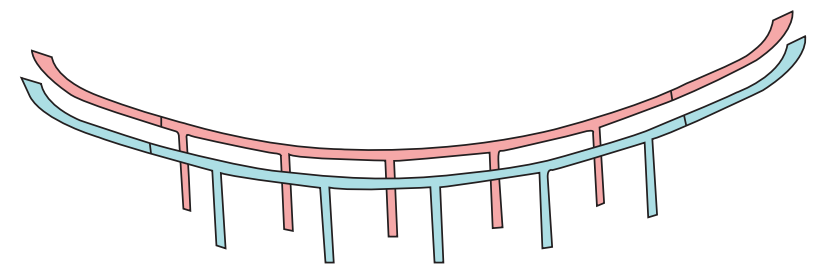

Figure 7: The selected monitoring planes (red color is return header and its branches, and blue color is supply header and its branches).

$$
\begin{aligned}
& \frac{\partial(\rho k)}{\partial t}+\frac{\partial\left(\rho k u_{i}\right)}{\partial x_{i}}=\frac{\partial}{\partial x_{j}}\left[\left(\mu+\frac{\mu_{t}}{\sigma_{k}}\right) \frac{\partial k}{\partial x_{j}}\right]+P_{k}-\rho\left(\varepsilon-\varepsilon_{0}\right)+S_{k} \\
& \frac{\partial(\rho \varepsilon)}{\partial t}+\frac{\partial\left(\rho \varepsilon u_{i}\right)}{\partial x_{i}}=\frac{\partial}{\partial x_{j}}\left[\left(\mu+\frac{\mu_{t}}{\sigma_{\varepsilon}}\right) \frac{\partial \varepsilon}{\partial x_{j}}\right]+\frac{1}{T_{e}} C_{1 \varepsilon} P_{\varepsilon}-C_{2 \varepsilon} f_{2} \rho\left(\frac{\varepsilon}{T_{e}}-\frac{\varepsilon_{0}}{T_{0}}\right)+S_{\varepsilon},
\end{aligned}
$$

where $t$ represents the flow time; $k$ represents the turbulent kinetic energy; $\varepsilon$ represents the dissipated energy; $u_{i}$ represents the time-averaged velocity; $\mu_{t}$ represents the turbulent viscosity; $f_{2}$ is the damping function; $T_{e}$ represents the large-eddy time scale; $T_{0}$ represents the source-dependent specific time scale; $P_{\varepsilon}$ and $P_{k}$ are the turbulence production terms; $\varepsilon_{0}$ corresponds to the ambient turbulence value in the source; $C_{1 \varepsilon}, C_{2 \varepsilon}, \sigma_{\varepsilon}$, and $\sigma_{k}$ represent the model coefficients; and $S_{k}$ and $S_{\varepsilon}$ represent the source term.

3.3. Meshing. FLUENT-Meshing is adopted for the meshing of the model [15]. An unstructured tetrahedral mesh was mainly generated in this study. Five layers of mesh were built for the near-wall boundary. The validation of grid independence is an essential step for FLUENT simulation. In this study, four mesh types were used for the validation, namely, 1.5 million, 3.3 million, 5.6 million, and 9.0 million meshes. The outlet velocity of the Z-type manifold is treated as the objective parameter. Figure 8 shows the temperature value with different mesh numbers. According to the results, a 3.3 million mesh can be regarded as a grid-independent solution [24, 25].

\section{Results and Discussions}

Figure 9 shows the change in the water volume fraction of the U-type and Z-type manifolds over time when the pressure difference was $5 \mathrm{kPa}, 10 \mathrm{kPa}$, and $20 \mathrm{kPa}[26,27]$. In addition, it could be seen from these figures that the water in the U-type and Z-type manifolds could be completely blown out under a pressure difference of $20 \mathrm{kPa}$ and could not be completely blown out under a pressure difference of $5 \mathrm{kPa}$. However, the amount of residual water in the Z-type manifold was less than that in the U-type manifold. Furthermore, under a pressure difference of $10 \mathrm{kPa}$, the water in the Z-type manifold was completely drained, but there was little water trapped in the U-type manifold. Meanwhile, there was a significant difference in the volume fraction of the liquid phase at $3 \mathrm{~s}$. The contour of the water volume fraction in the U-type and Z-type manifolds at $3 \mathrm{~s}$ is presented in Figure 10. Moreover, it was observed that the amount of water in the Z-type manifold was less than that in the U-type manifold at the same time. The water was mainly trapped in the location far away from the inlet of the supply header. This location was also called the water retention domain.

According to the theoretical analysis, the discharge speed of the branch pipe is related to the static pressure difference between the manifold intake and exhaust. Therefore, Figure 11 shows the static pressure values of the U-type manifold and the Z-type manifold under the monitoring surface shown in Figure 7 at $3 \mathrm{~s}$. Through comparative theoretical analysis, it is found that the pressure of the manifold intake is mainly distributed in shadow area 2, showing a decreasing trend to a stable value and then a rising trend, and the friction resistance term and inertia term are 


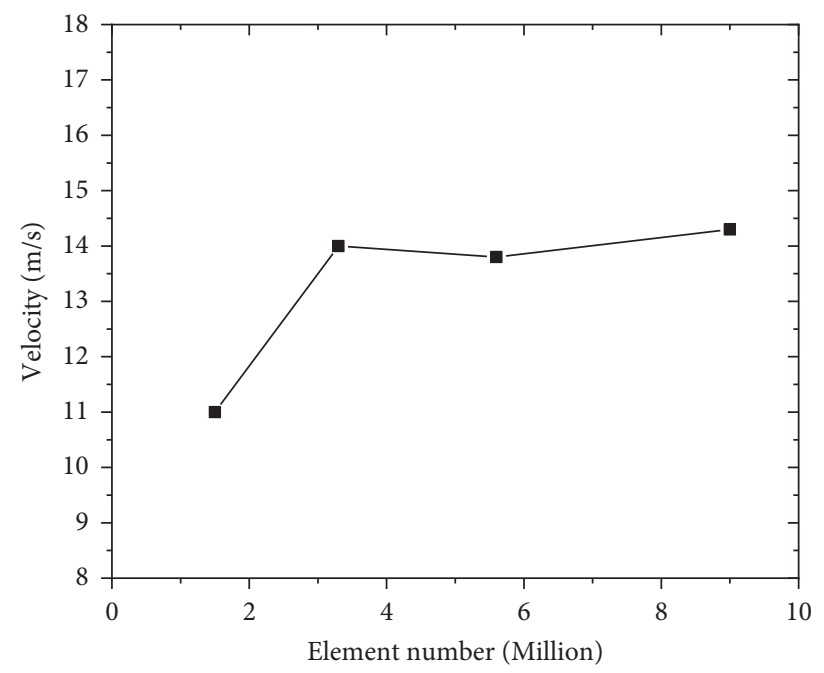

Figure 8: The velocity value with different mesh numbers.

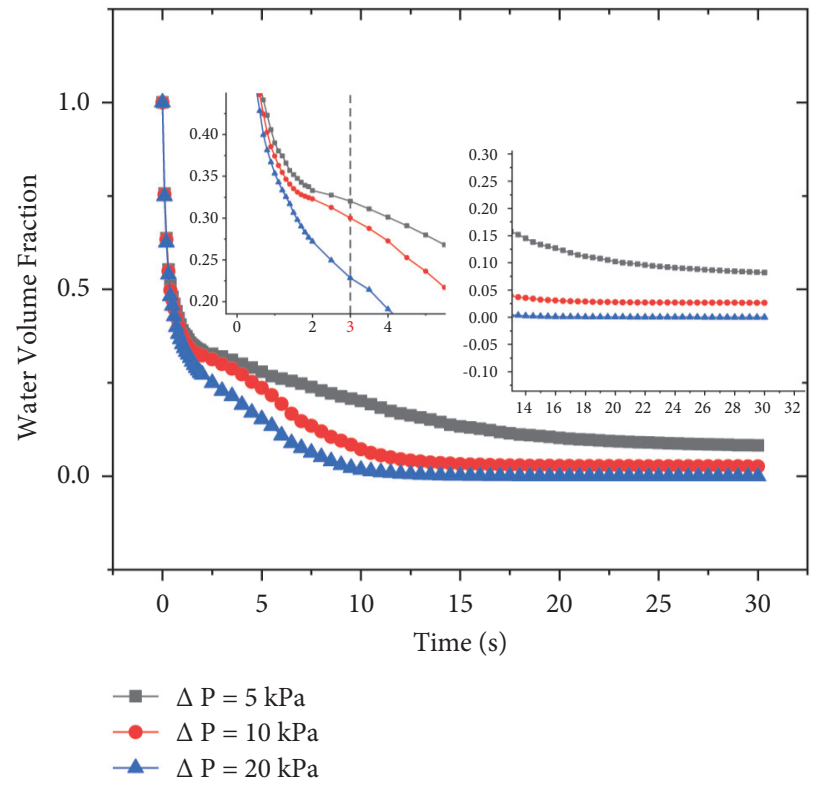

(a)

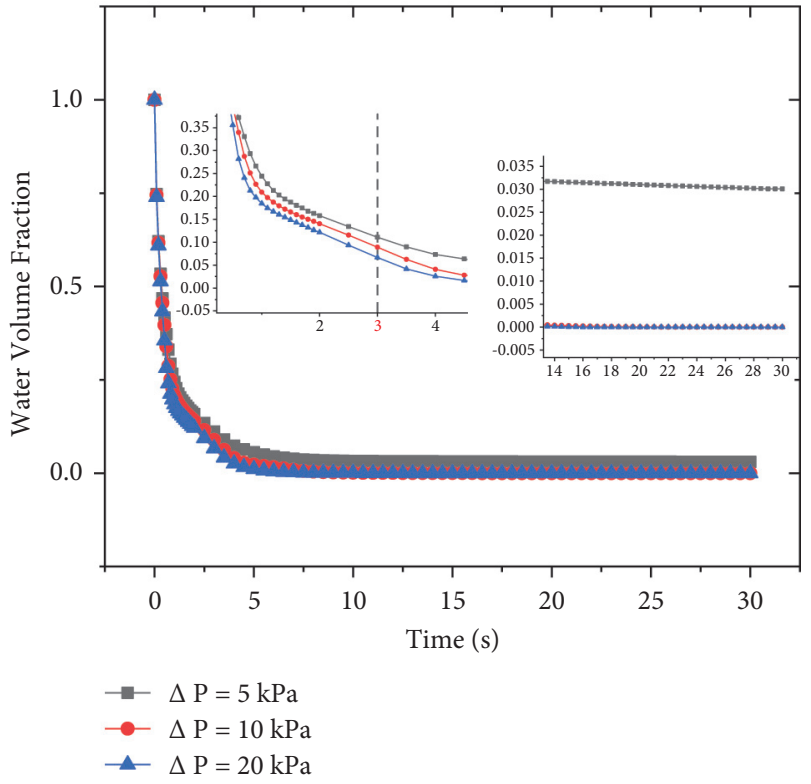

(b)

FiguRE 9: Variation in liquid volume fraction with time under different pressure differences. (a) U-type manifold. (b) Z-type manifold.

restrained by each other. Under the effect of fluid collection, the fluid kinetic energy increases and the static pressure decreases along the flow direction of the manifold exhaust, but the outlet directions of the U-type manifold and the Z-type manifold are in the opposite position, resulting in the pressure of the manifold exhaust of the U-type manifold gradually increasing along the positive $X$ direction while the pressure of the manifold exhaust of the Z-type manifold gradually decreasing along the positive $X$ direction. In addition, when there is no retained water in the branch pipe, the flow rate is large, resulting in a decrease in static pressure. The blocking effect of the retained water in the branch pipe on the flow rate leads to a decrease in the flow rate and an increase in the static pressure. Figure 11 shows that the static pressure of branch pipes 4 and 5 on the right side of the U-type manifold and the Z-type manifold increases at $3 \mathrm{~s}$. Compared with the gas-liquid distribution in Figure 10, it is found that there is retained water in the right branch pipe of the U-type manifold and the Z-type manifold, which belongs to the water retention area. Due to a large amount of retained water, the drainage resistance of the branch pipe is large. Compared with the left branch pipe of the manifold, the larger drainage resistance of the right branch pipe needs to be matched with a larger static pressure difference, but the static pressure difference in the water retention area of the U-type manifold is small. At this time, according to a comparison of the static pressure distribution of the Z-type manifold intake and exhaust at $3 \mathrm{~s}$, it is found that the static 

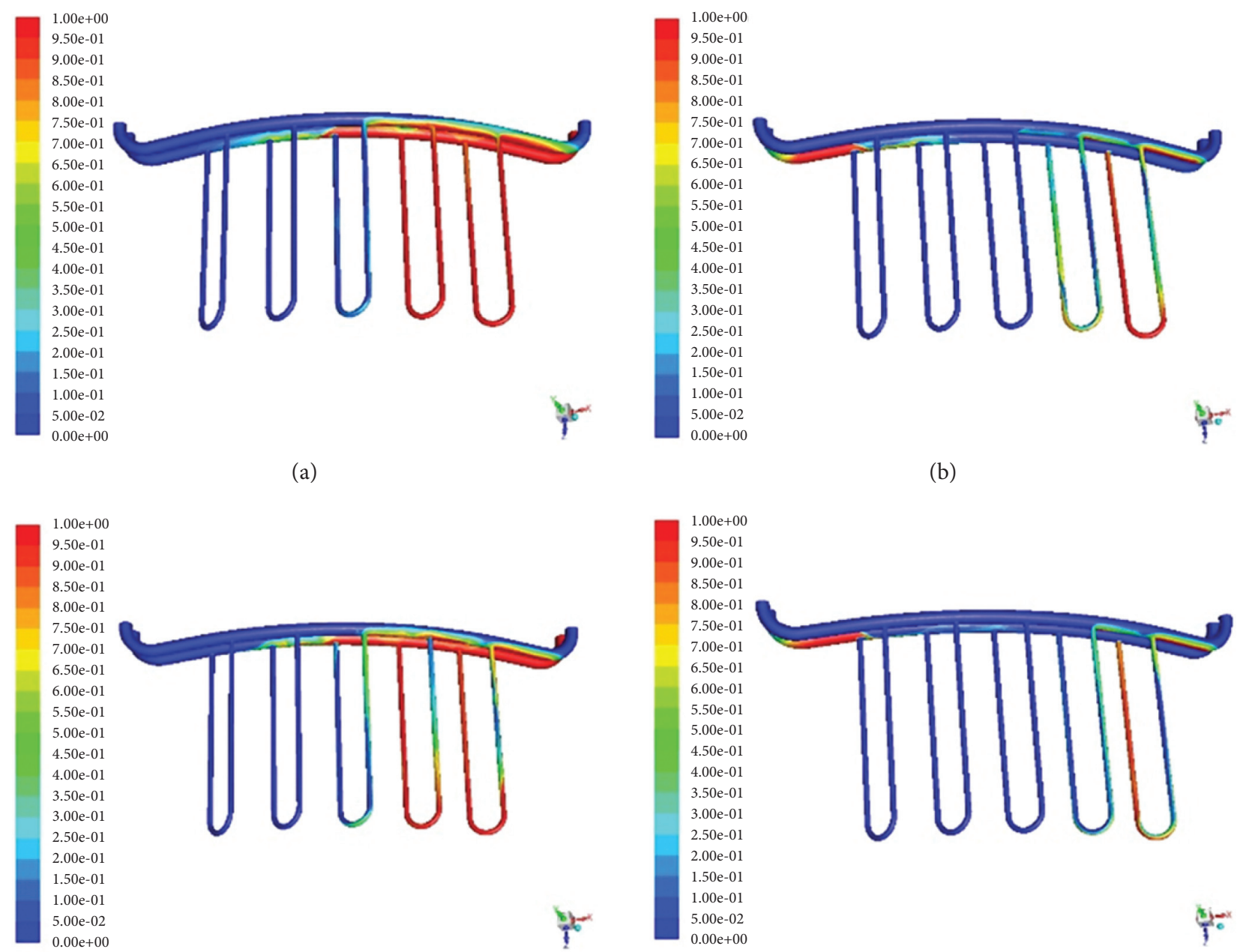

50e- 01

$2.00 \mathrm{e}-01$
$1.50 \mathrm{e}-01$

$100 \mathrm{e}-01$

$5.00 \mathrm{e}-02$
$0.00 \mathrm{e}+00$
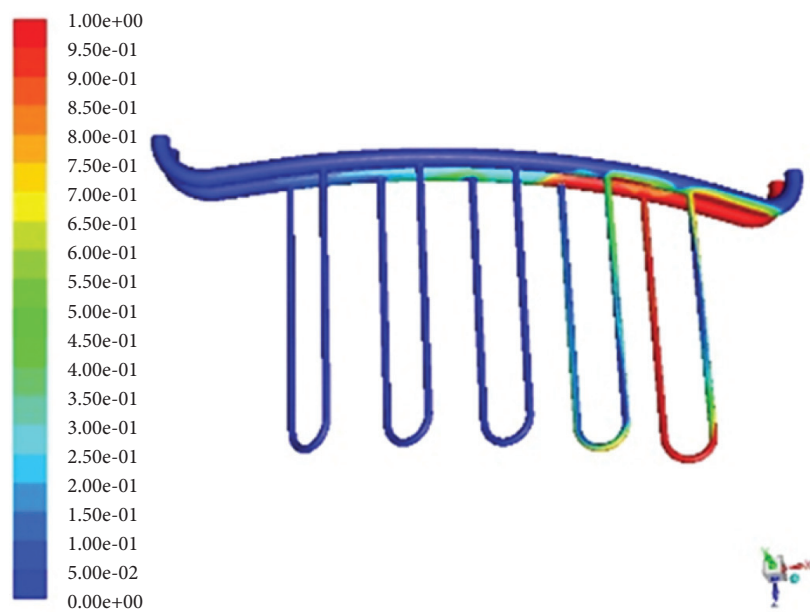

$1.50 \mathrm{e}-01$

$0.00 \mathrm{e}+00$

(a)

(b)

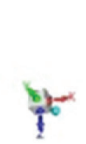

(c)

(d)

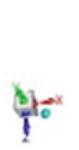

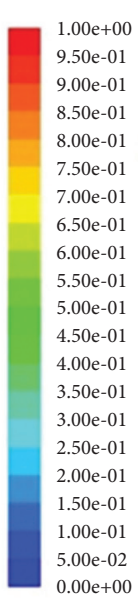

$1.00 \mathrm{e}+00$ $50 \mathrm{e}-01$ . $00 \mathrm{e}-01$ 7.5e-01 $50 \mathrm{e}-01$ $6.00 \mathrm{e}-01$ $.50 \mathrm{e}-01$ $00 \mathrm{e}-01$

.50e- 01 $.00 \mathrm{e}-01$ e0e- 01 (1)

(

$1.00 \mathrm{e}+00$ $9.50 \mathrm{e}-01$ $9.00 \mathrm{e}-01$ $8.50 \mathrm{e}-01$ $8.00 \mathrm{e}-01$ $8.00 \mathrm{e}-01$
$7.50 \mathrm{e}-01$
7.01 $7.00 \mathrm{e}-01$

$6.50 \mathrm{e}-01$ $6.00 \mathrm{e}-01$ $6.00 \mathrm{e}-01$
$5.50 \mathrm{e}-01$ $5.00 \mathrm{e}-01$ $4.50 \mathrm{e}-01$ $4.50 \mathrm{e}-01$
$4.00 \mathrm{e}-01$ $3.50 \mathrm{e}-01$ $3.00 \mathrm{e}-01$ $2.50 \mathrm{e}-01$ 2.00e-01 $1.50 \mathrm{e}-01$

$1.00-01$

$5.00 \mathrm{e}-02$ $0.00 \mathrm{e}+00$
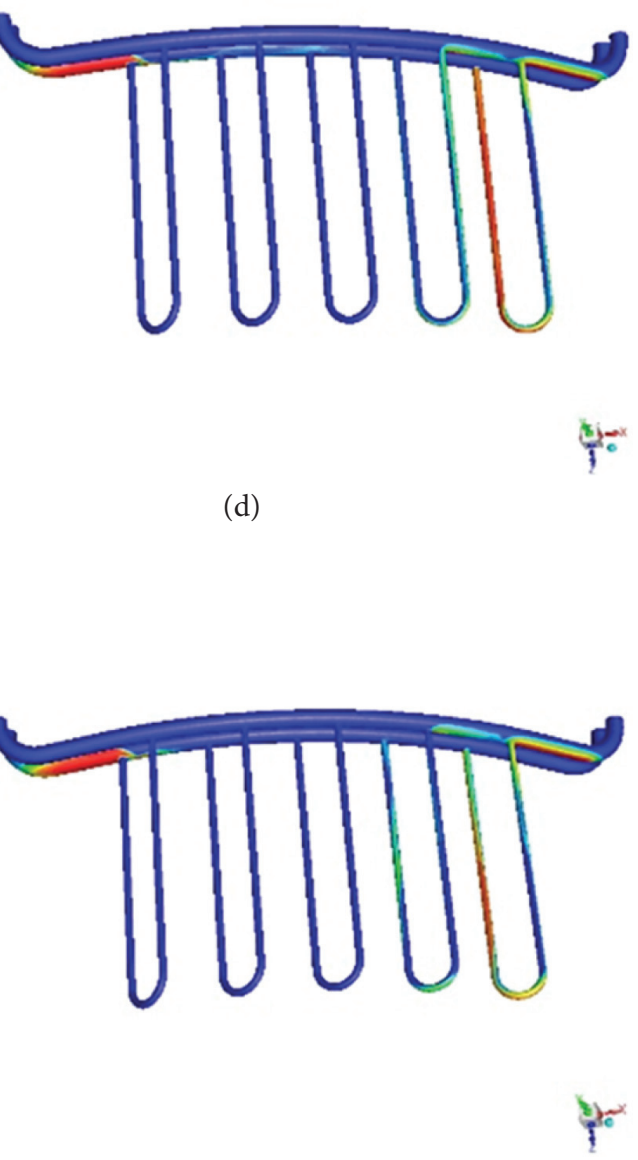

(e)

Figure 10: The contour of the water volume fraction at $3 \mathrm{~s}$. (a) $5 \mathrm{kPa}$ pressure difference in the $\mathrm{U}$ type. (b) $5 \mathrm{kPa}$ pressure difference in the $\mathrm{Z}$ type. (c) $10 \mathrm{kPa}$ pressure difference in the $\mathrm{U}$ type. (d) $10 \mathrm{kPa}$ pressure difference in the $\mathrm{Z}$ type. (e) $20 \mathrm{kPa}$ pressure difference in the $\mathrm{U}$ type. (f) $20 \mathrm{kPa}$ pressure difference in the $\mathrm{Z}$ type. 


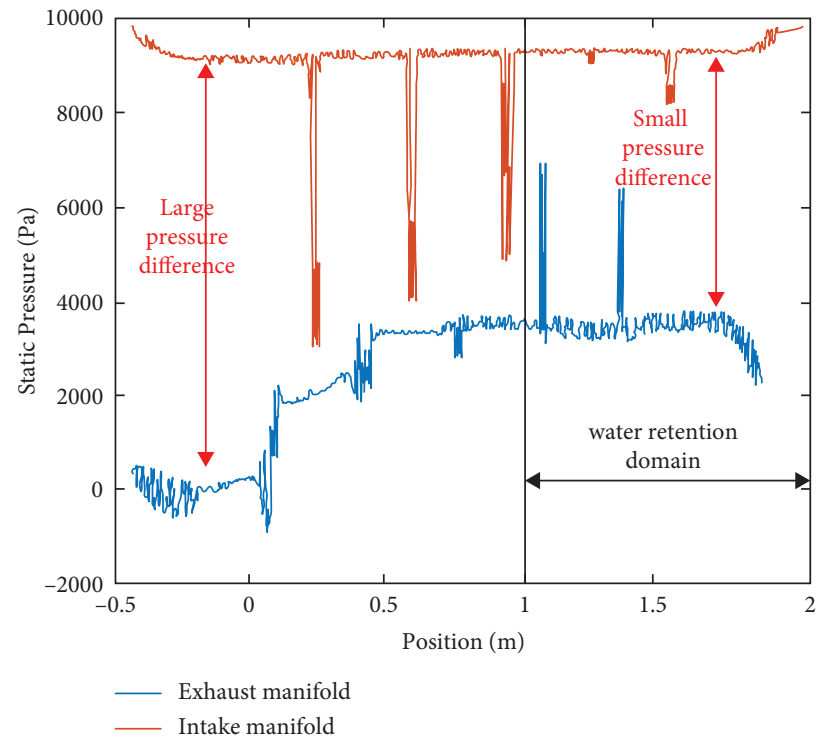

(a)

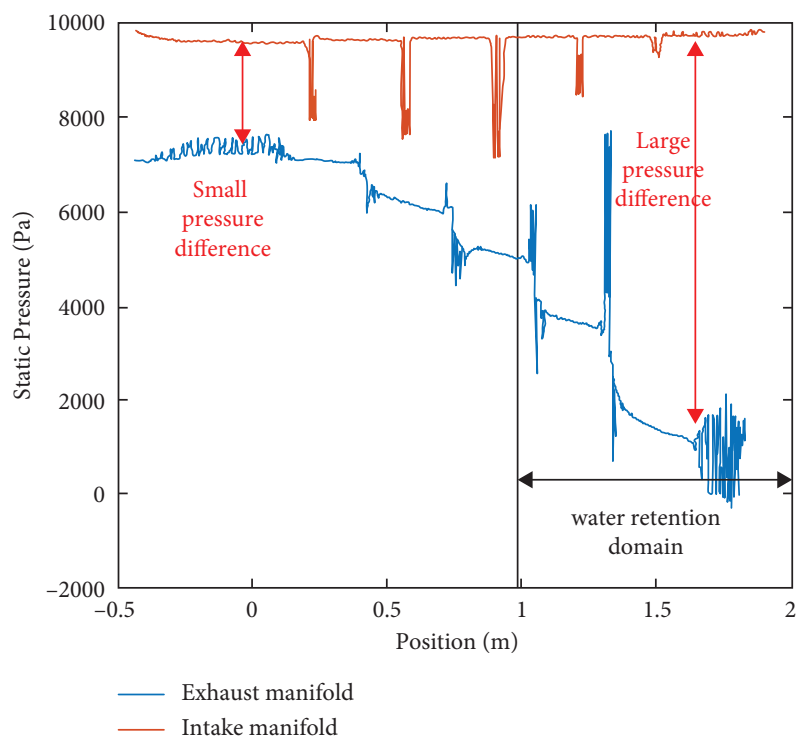

(b)

FIgURe 11: Pressure distribution of the manifold monitoring planes at 3 s. (a) U-type manifold. (b) Z-type manifold.

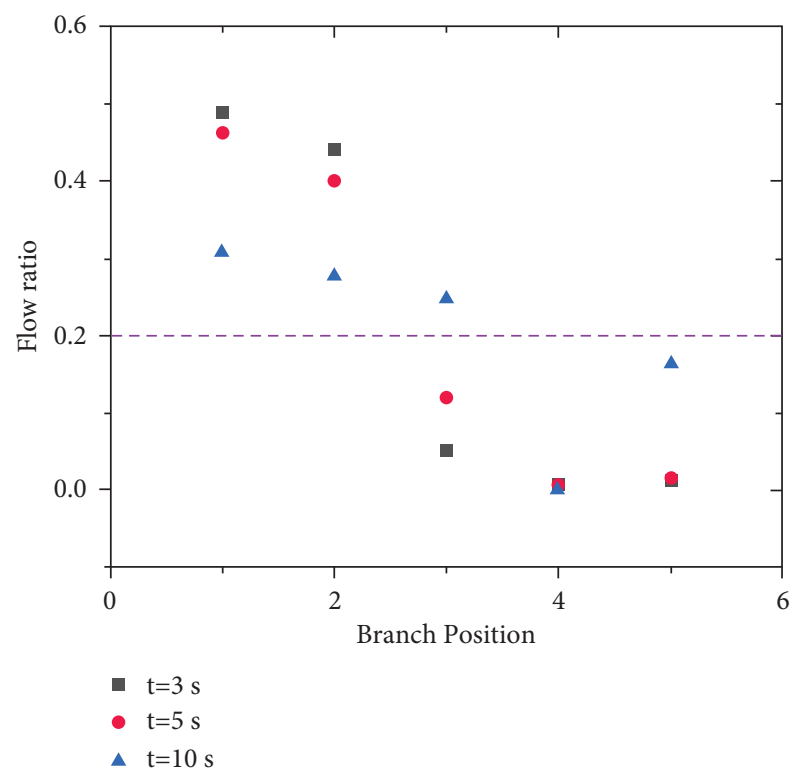

(a)

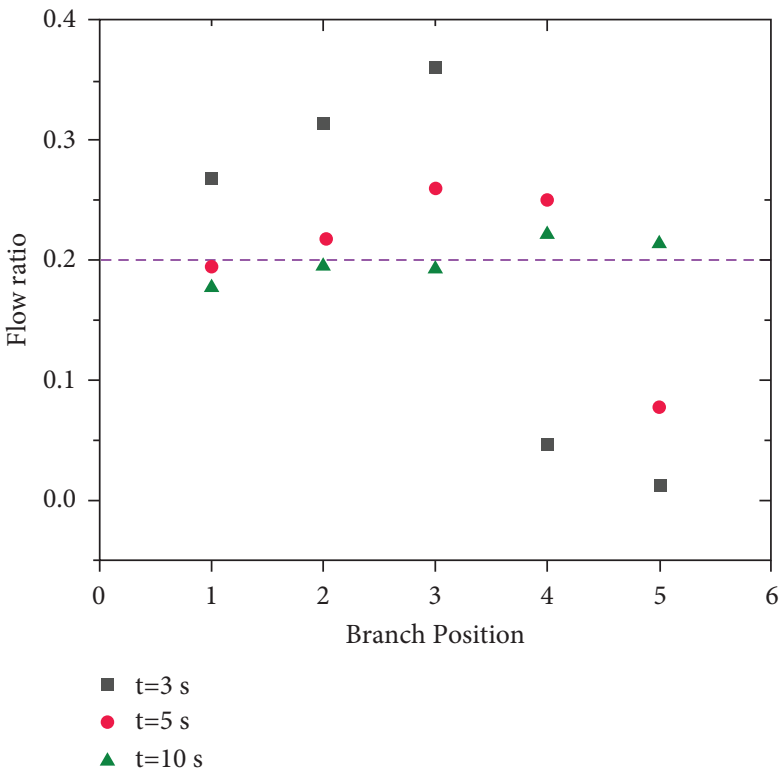

(b)

FIGURE 12: Flow ratio distribution of the manifold branches under a pressure difference of $10 \mathrm{kPa}$. (a) U-type manifold. (b) Z-type manifold.

pressure difference of the Z-type manifold in the water retention area is large. Even if the branch pipe has a large resistance, the retained water can be discharged well under large differential pressure, so it has a better drainage effect and better dryness.

Due to the varied pressure difference and resistance distribution in the manifold, there was a phenomenon of uneven flow in the branch pipe of the manifold. Therefore, the flow ratio, which was defined in equation (22), is an important indicator to predict the uniformity of branch flow based on U-type and Z-type manifolds. The value of the flow ratio is between 0 and 1 . The closer the flow ratio is to 0.2 , the more uniform the flow distribution will be.

$$
\gamma_{i}=\frac{Q_{i}}{\sum_{i=1}^{N} Q_{i}} .
$$

In equation (22), " $i$ " denotes the locations of the branches of the manifolds. Thus, $Q_{i}$ refers to the gas-liquid mixed flow at location $i$.

At different points of time, when the pressure difference was $10 \mathrm{kPa}$, the flow ratio of the U-type and Z-type 


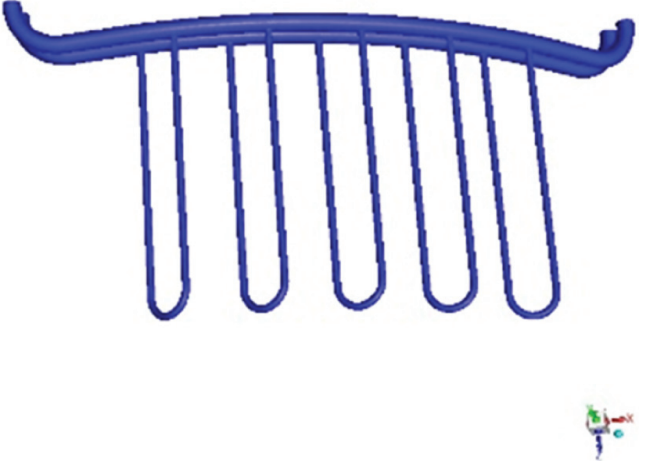

(a) (b)

Figure 13: The contour of the water volume fraction at $30 \mathrm{~s}$. (a) U-type manifold. (b) Z-type manifold.

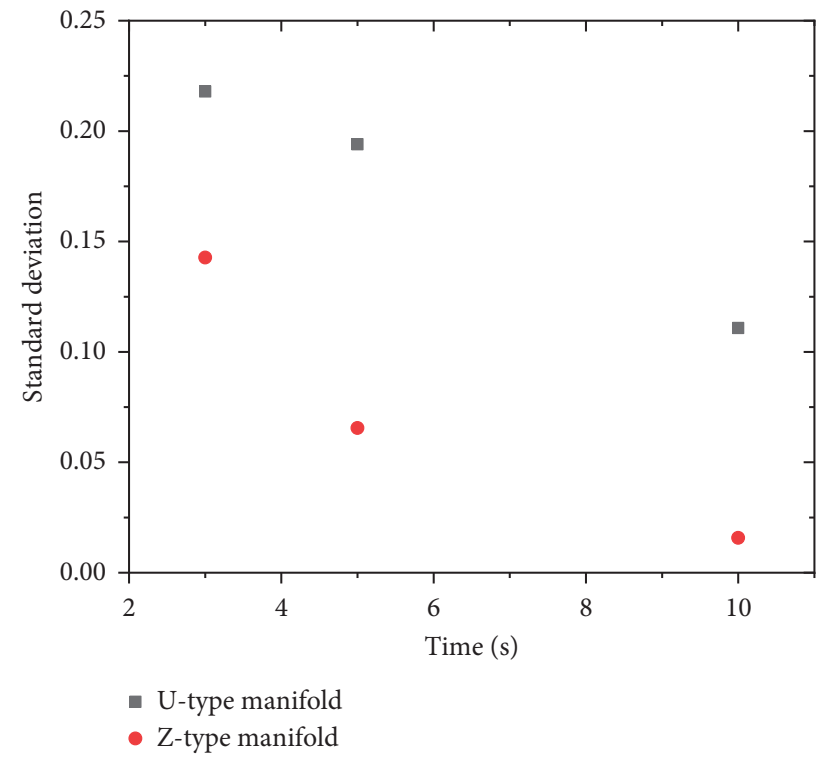

Figure 14: Flow ratio standard deviation distribution at different time points.

manifold branches is shown in Figure 12. From Figure 12, it was observed that the flow ratio of the branches located in the water retention domain was far below 0.2 . However, the flow ratio of each branch of the Z-type manifold is more uniform than that of the U-type manifold over time. Moreover, due to the large pressure difference in the Z-type manifold water retention domain, the flow ratio of branches 4 and 5 was slightly higher than 0.2 . Furthermore, the no. 4 branch flow ratio of the U-type manifold was nearly 0 , which indicated that the water could be trapped in the no. 4 branch. This could be verified by the contour of the water volume fraction at $30 \mathrm{~s}$, as shown in Figure 13. The water in the Z-type manifold had been completely drained, but residual water still stayed in the no. 4 branch of the U-type manifold.
The flow ratio in the branches of the U-type and Z-type manifolds was uniform, but there were differences. Figure 14 shows the flow ratio standard deviation distribution of different manifold branches at different time points. The closer the value is to zero, the more uniform the flow distribution is. It is further found that the flow distribution of Z-type manifold branches is always more uniform than that of U-type manifold branches. Furthermore, the branch nonuniform extent of the U-type manifold was always more than ten percent. On the other hand, the nonuniform extent of the branches of the Z-type manifold was already less than ten percent at $5 \mathrm{~s}$ and almost uniformly distributed at $10 \mathrm{~s}$. Consequently, compared with the U-type manifold, the branch flow distribution of the Z-type manifold is more uniform under the condition of drainage. 


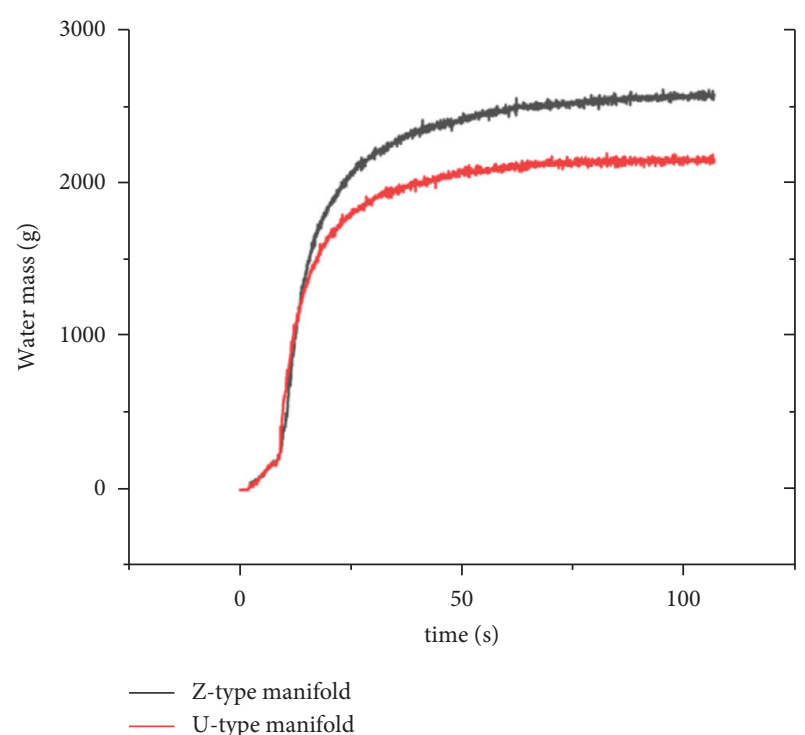

FIGURE 15: Comparison of displacement of different manifold types.

\section{Validation of Simulation Results against Selected Boundary Conditions}

To validate the simulation results for the effect of the U-type and Z-type manifolds on the drainage drying process, geometric model test samples consistent with the simulation are prepared. The differential pressure transmitter is used to measure the inlet and outlet pressures of the manifold test samples to reach the selected differential pressure boundary condition of $10 \mathrm{kPa}$. At the same time, the drainage discharge of the manifolds in the drainage and drying processes is measured in real time by the weighing sensor. The drainage discharge of different types of manifolds is compared under the selected boundary conditions.

Based on the experimental results, both amounts of water draining of the U-type and Z-type manifolds are shown in Figure 15. Under the same selected boundary conditions, the drainage discharge of the Z-type manifold is greater, which again verifies that the $Z$-type manifold has more advantages under the conditions of drainage and drying. The experimental results are reliable and in line with expectations of the theoretical and numerical analysis.

\section{Conclusions}

This study uses dynamic theory to construct a branch pipe drainage characteristic model and a pressure distribution model of manifold intake and exhaust and to study the effect of manifold type on the drying process. Based on numerical analysis of the drying process of the U-type and Z-type manifolds, taking the volume fraction of the liquid phase in the manifolds, the pressure distribution of the manifold intake and exhaust, and the flow distribution of the branch pipe as indicators, it is proposed that the Z-type manifold can obtain higher dryness at the same pressure difference, which is verified by comparing the experimental value of drainage discharge. Moreover, the influence of the manifold structure on the drying system is revealed, which provides a reference for the design of fusion reactor drying systems in the future.

\section{Data Availability}

The research data used to support the findings of this study are available from the corresponding author upon request.

\section{Conflicts of Interest}

The authors declare that they have no conflicts of interest.

\section{Acknowledgments}

The research was supported by the Hefei Institute of Materials Science, Chinese Academy of Sciences (no. YZJJ2020QN15).

\section{References}

[1] Z. Miao and T. Xu, "Single phase flow characteristics in the headers and connecting tube of parallel tube platen systems," Applied Thermal Engineering, vol. 26, no. 4, pp. 396-402, 2006.

[2] J. Wang, "Pressure drop and flow distribution in parallelchannel configurations of fuel cells: U-type arrangement," International Journal of Hydrogen Energy, vol. 33, no. 21, pp. 6339-6350, 2008.

[3] M. S. Gandhi, A. A. Ganguli, J. B. Joshi, and P. K. Vijayan, "CFD simulation for steam distribution in header and tube assemblies," Chemical Engineering Research and Design, vol. 90, no. 4, pp. 487-506, 2012.

[4] H. Asgharian, E. Afshari, and E. Baniasadi, "Numerical investigation of pressure drop characteristics of gas channels and $U$ and $Z$ type manifolds of a PEM fuel cell stack," International Journal of Energy Research, vol. 44, no. 7, pp. 5866-5880, 2020.

[5] O. K. Siddiqui, "Flow distribution in U-type and Z-type manifolds: experimental and numerical investigation," Arabian Journal for Science and Engineering, vol. 45, pp. 60056020, 2020.

[6] C. Vallée, T. Höhne, H.-M. Prasser, and T. Sühnel, "Experimental investigation and CFD simulation of horizontal stratified two-phase flow phenomena," Nuclear Engineering and Design, vol. 238, no. 3, pp. 637-646, 2008.

[7] M. K. Bassiouny and H. Martin, "Flow distribution and pressure drop in plate heat exchanges. Part I, U-type arrangement," ences, vol. 39, no. 4, pp. 969-976, 2020.

[8] J. Wang, "Pressure drop and flow distribution in parallelchannel configurations of fuel cells: Z-type arrangement," International Journal of Hydrogen Energy, vol. 35, no. 11, pp. 5498-5509, 2010.

[9] G. B. Wallis, Annular flow in One-Dimensional Two-phase Flowpp. 315-367, McGraw, MI, USA,, 1st. edition, 1969.

[10] J. Wang, G. H. Priestman, and D. Wu, "A theoretical model of uniform flow distribution for the admission of high-energy fluids to a surface steam condenser," Journal of Engineering for Gas Turbines and Power, vol. 123, no. 2, pp. 472-475, 2001. 
[11] J. Wang, Z. Gao, G. Gan, and D. Wu, "Analytical solution of flow coefficients for a uniformly distributed porous channel," Chemical Engineering Journal, vol. 84, no. 1, pp. 1-6, 2001.

[12] R. A. Bajura, "A model for flow distribution in manifolds," Journalof Engineering for Gas Turbines and Power, vol. 93, no. 4, pp. 7-12, 1971.

[13] J. Wang, "Flow distribution in U-type layers or stacks of planar fuel cells," Journal of Power Sources, vol. 190, no. 2, pp. 511-512, 2009.

[14] J. Y. Wang, X. L. Ge, and D. D. Wu, "Process of flow in manifolds," Journal of Advanced Mechanical Design, Systems, and Manufacturing, vol. 28, no. 3, pp. 392-401, 1998.

[15] P. I. Shen, "The effect of friction on flow distribution in dividing and combining flow manifolds," Journal of Fluids Engineering, vol. 114, no. 1, pp. 121-123, 1992.

[16] Y. Shi, Study on Medium Distribution Mechanism and Flow Uniform Distribution Strategy of Parallel Connection Pipes for Liquefied Natural Gas Cooling Box, DS dissertation, Deptment of Mechanical Engineering, Shandong University, China, Shandong, 2018.

[17] A. A. Haerter, "Flow distribution and pressure change along slotted or branched ducts," Journal of ASHRAE, vol. 5, no. 1, pp. 47-59, 2006.

[18] W. X. Bi, J. Y. Li, and Z. J. Lin, "Flow uniformity optimization for large size planar solid oxide fuel cells with U-type parallel channel design," Journal of Power Sources, vol. 115, no. 1, pp. 54-65, 2003.

[19] J. Z. Jiang, W. M. Zhang, and Z. Y. Li, "Mathematic simulation on gas-liquid two-phase flow of pipe pigging by gas condition," Journal of System Simulation, vol. 25, no. 2, pp. 383388, 2013.

[20] W. Q. Yuan and S. Liu, "Transient numerical modeling of Gas-liquid two-phase flow in water-filling pipelines," Journal of Tongji University, vol. 38, no. 5, pp. 709-715, 2010.

[21] W. Li, J. Hu, P. Fu et al., "Numerical study on water draining process pushed by nitrogen in EAST upper divertor," Fusion Engineering and Design, vol. 170, no. 6, Article ID 112545, 2021.

[22] R. Guo, W. M. Zhang, J. Z. Jiang, J. Li, and Y. T. Yuan, "Gas-liquid flow characteristics in evacuation process for ascending pipelines," Journal of China University of Petroleum, vol. 41, no. 5, pp. 146-151, 2017.

[23] R. Y. Bai, J. W. Bao, L. Song, H. D. Li, and Y. H. Liu, "Transient numerical simulation analysis of gas-liquid two-phase flow under the condition of water filling and exhaust in municipal water supply pipeline," Journal of Inner Mongolia University of Technology (Social Sciences Edition), vol. 36, no. 3, pp. 222-228, 2017.

[24] G. Binbin and C. Jinping, Three-dimensional structure grid generation method in ANSYS ICEM CFDpp. 153-205, China Water \& Power Press, Beijing, China, 1st, edition, 2017.

[25] J. P. Tang, Post-processing method in ANSYS FLUENT 16.0 Super Learning Manualpp. 128-172, Posts and Telecom Press, Beijing, China, 1 st. edition, 2016.

[26] S. Kim, W. Van Hove, J. Ferrada et al., "Draining and drying process development of the Tokamak cooling water system of ITER," Fusion Engineering and Design, vol. 109-111, no. 6, pp. 272-277, 2016.

[27] M. Mohammadi, "Numerical study flow uniformity and pressure characteristics within a microchannel array with triangular manifolds," Computer and chemical Engineering, vol. 52, Article ID 423, 2012. 